\title{
RNA in Defense: CRISPRs Protect Prokaryotes against Mobile Genetic Elements
}

\section{Matthijs M. Jore, Stan J.J. Brouns, and John van der Oost}

Laboratory of Microbiology, Wageningen University, Netherlands

Correspondence: John.vanderoost@wur.nl

\section{SUMMARY}

The CRISPR/Cas system in prokaryotes provides resistance against invading viruses and plasmids. Three distinct stages in the mechanism can be recognized. Initially, fragments of invader DNA are integrated as new spacers into the repetitive CRISPR locus. Subsequently, the CRISPR is transcribed and the transcript is cleaved by a Cas protein within the repeats, generating short RNAs (crRNAs) that contain the spacer sequence. Finally, crRNAs guide the Cas protein machinery to a complementary invader target, either DNA or RNA, resulting in inhibition of virus or plasmid proliferation. In this article, we discuss our current understanding of this fascinating adaptive and heritable defense system, and describe functional similarities and differences with RNAi in eukaryotes.

\section{Outline}

1 Introduction

2 CRISPR loci and Cas genes

3 Mode of action
4 Analogy with RNAi in eukaryotes

5 Concluding remarks

References

Editors: John F. Atkins, Raymond F. Gesteland, and Thomas R. Cech

Additional Perspectives on RNA Worlds available at www.cshperspectives.org

Copyright (C 2012 Cold Spring Harbor Laboratory Press; all rights reserved; doi: 10.1101/cshperspect.a003657

Cite as Cold Spring Harb Perspect Biol 2012;4:a003657 


\section{INTRODUCTION}

The evolution of micro-organisms is significantly influenced both qualitatively and quantitatively by the continuous exchange of genomic material with mobile genetic elements: viruses and plasmids. Viruses are among the most abundant entities on earth (Bergh et al. 1989; Wommack and Colwell 2000) and they proliferate by a series of events: adsorption of the virion to the host's cell wall, injection of the viral genome (DNA, RNA) through the cell membrane(s), expression of viral genes, replication of the viral genome and assembly of viral protein capsids, and finally release of progeny virions (Sturino and Klaenhammer 2004). Plasmids are another main class of selfish mobile elements. After entry, plasmid DNA resides in a host, either free in the cytoplasm or as integrated sequence in the host genome. Plasmids can be transferred from donor to recipient via conjugation, making use of dedicated transfer systems (Llosa et al. 2002).

Despite the occasional gain of function as a result of horizontal gene transfer, recombination with mobile elements can also cause severe damage (disruption of either structural or regulatory regions on the host genome leads to loss of function). Additionally, phage infections can eventually lead to host cell lysis. To avoid these detrimental effects, sophisticated mechanisms have evolved to defend host organisms against nucleic acids of invading mobile elements. Several defense systems have been recognized in prokaryotes that are very different from the eukaryotic immune systems. A passive defense mechanism may act at the level of virion adsorption and/or injection of its genomic material. Spontaneous mutations in virus receptor proteins of the host can perturb virus attachment and genome injection, not affecting host fitness, for example, the maltoporin of Escherichia coli used by phage lambda (Hofnung et al. 1976). A well known active defense mechanism is the restriction-modification (R-M) system. Dedicated methyltransferases modify potential cleavage sites of the host DNA, preventing strand cleavage by restriction enzymes. Incoming invader DNA lacks these modifications, and is therefore a target for digestion by these endonucleases (Review, Tock and Dryden 2005). An additional mechanism that appears functionally analogous to eukaryotic apoptosis is the prokaryotic Abortive infection mechanism (Abi). This mechanism inhibits phage multiplication either by blocking the phage replication machinery, or by inhibiting host translation. This results in death of both host and virus, a sacrifice that will save the rest of the population (Chopin et al. 2005).

Recently, another defense mechanism has been discovered that is based on clusters of regularly interspersed short palindromic repeats (CRISPRs) and CRISPR-associated genes (cas genes). The CRISPR/Cas system can integrate nucleic acid fragments from invading mobile elements into the CRISPR locus. The CRISPR is transcribed and cleaved into short mature RNAs (crRNAs). These crRNAs specifically guide the Cas protein machinery to their complementary targets: either DNA or RNA from invading viruses or plasmids. Thus, the CRISPR/Cas system can provide the host with acquired and heritable resistance (reviewed in Sorek et al. 2008; van der Oost et al. 2009; Horvath and Barrangou 2010; Karginov and Hannon 2010; Marraffini and Sontheimer 2010a). In this article, we describe mechanistic features of the CRISPR/Cas system, and we discuss the similarities and differences with RNA interference in eukaryotes.

\section{CRISPR LOCI AND CAS GENES}

CRISPRs were first discovered in 1987 when a chromosomal fragment from E. coli K12 was sequenced (Ishino et al. 1987). Since then, many CRISPR sequences have been identified in prokaryotic genomes (for overview, see: http://crispr.u-psud.fr/crispr/CRISPRdatabase.php). CRISPRs have been detected in $48 \%$ of the sequenced bacterial genomes and in $95 \%$ of the sequenced archaeal genomes. CRISPRs are composed of a cluster of identical repetitive sequences that are separated by nonidentical spacer sequences of similar length (see a later discussion). The CRISPR array is often preceded by an AT-rich leader sequence of up to 500 base pairs (bp) (Jansen et al. 2002). The number of CRISPR loci per genome ranges from one to 20, varying in length from a few to hundreds of repeat-spacer pairs. The present record holder is a CRISPR of Chloroflexus sp. with 374 repeats and spacers. Twelve major types of CRISPR have been proposed, based on sequence similarity of the repeats (Kunin et al. 2007). The repeat size varies from 24 to $47 \mathrm{bp}$, whereas the spacer size ranges from 24 to $72 \mathrm{bp}$. The size of repeats and spacers are typically around $30 \mathrm{bp}$. Some repeats have palindromic sequences that encode CRISPR RNAs with potentially strong secondary structures, whereas other sequences appear to lack such structures (Fig. 1B). Each cluster correlates mainly with one Cas subtype, as discussed later. In 2005 , three different research groups independently observed that at least a subset of the spacer sequences are identical to phage and plasmid DNA sequences (Bolotin et al. 2005; Mojica et al. 2005; Pourcel et al. 2005). The virus or plasmid fragment matching the spacer sequence is called the proto-spacer (Deveau et al. 2008). The observation that spacer sequences were derived from viral sequences has led to the hypothesis that the CRISPR/Cas system might be involved in prokaryotic resistance to alien nucleic acids (Reviewed, Makarova et al. 2006). The composition of 
A

S. epidermidis (Csm)

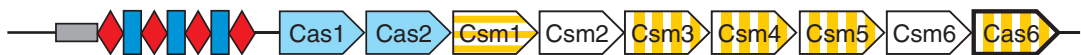

E. coli (Cse)

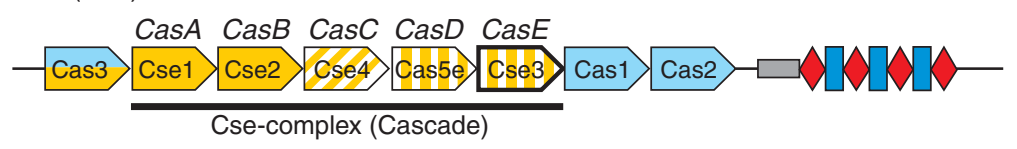

P. furiosus (Cst $+\mathrm{Cmr}$ )

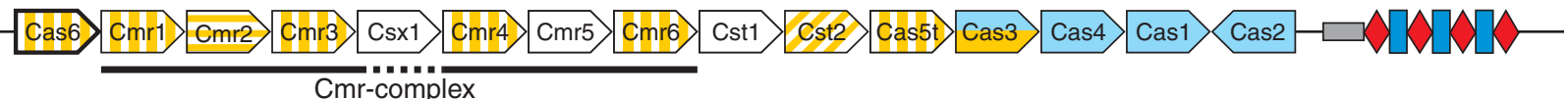

S. thermophilus (Csn)

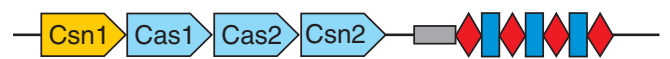

B

S. epidermidis

E. coli

P. furiosus

S. thermophilus

C

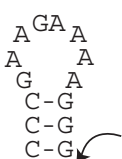

5'-GGAUCGAUACCCAC-GACGAGAAC - 3'

S. epidermidis
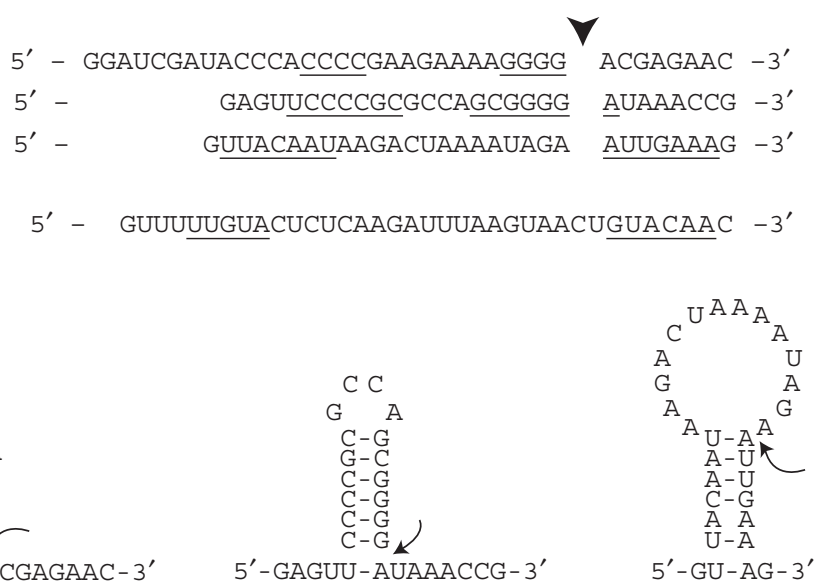

5'-GAGUU-AUAAACCG-3'

E. coli

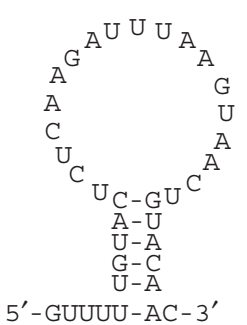

S. thermophilus

Figure 1. Overview of the four CRISPR/Cas subtypes that are described in this article. For an overview of all eight CRISPR/Cas subtypes, see Haft et al. 2005 and van der Oost et al. 2009. (A) cas gene neighborhoods in four experimentally studied organisms, each representing a different subtype indicated between brackets. CRISPRs consist of a leader (grey box), repeats (red diamonds), and spacers (blue boxes). Only a fragment of the CRISPR is shown. Genes are indicated as arrows. Blue arrows indicate genes that are (possibly) involved in spacer acquisition. Yellow arrows indicate genes that are involved in CRISPR transcription and processing and target interference. The endonucleases that cleave pre-crRNA generating crRNA are highlighted as bold arrows. Hatching patterns indicate gene similarity: RAMP genes have vertical lines, polymerase genes have horizontal lines, CasC homologs have diagonal lines, and other genes that are not related to each other are filled. Genes that encode proteins from isolated complexes (Cse-complex from E. coli and Cmr-complex from Pyrococcus furiosus) are underlined. (B) CRISPR RNA repeat sequences from each organism are given. The cleavage site is indicated by a triangle. Although the repeat sequences are different, all CRISPR RNA cleavage events generate an eight-nucleotide $5^{\prime}$ handle. Please note that the cleavage site in Streptococcus thermophilus CRISPR RNA has not been determined. Palindromic sequences are underlined. $(C)$ Predicted secondary structures of the different CRISPR RNA repeats. Cleavage sites are indicated with an arrow. As described previously by Kunin et al., the repeat of $P$. furiosus is not likely to form a stem loop (Kunin et al. 2007). 
the CRISPR is hypervariable and is rapidly shaped by extrachromosomal elements in the host's environment (Lillestol et al. 2006; Andersson and Banfield 2008; Tyson and Banfield 2008; Banfield and Young 2009; Held and Whitaker 2009; Lillestol et al. 2009). Extrachromosomal elements in turn respond by extensive gene shuffling (Andersson and Banfield 2008) or mutations (Deveau et al. 2008; Heidelberg et al. 2009; Semenova et al. 2009; van der Ploeg 2009) to escape the CRISPR defense mechanism, illustrating the ongoing battle between hosts and their predators.

A set of conserved cas genes can be found in close proximity of the CRISPR array. The encoded proteins were initially thought to be involved in DNA repair, because they had predicted nucleic acid related functions (Makarova et al. 2002). The link between cas genes and CRISPRs was made shortly thereafter (Jansen et al. 2002) and the four most conserved cas genes were identified. The cas gene products were further classified into $\sim 45$ distinct families (Haft et al. 2005). That number was later reduced to $\sim 25$ families (Makarova et al. 2006). The set of Cas proteins is composed of core proteins (Cas1-6), a diverse group of Repeat-Associated Mysterious Proteins (RAMPs), and more loosely associated Cas proteins, such as a polymerase. Based on the composition of the cas operons, eight Cas subtypes (Csa, Csd, Cse, Csh, Csm, Csn, Cst, and Csy) by Haft et al. (2005), or seven Cas systems (CASS1-7) by Makarova et al. (2006), have been proposed that each contain a certain set of core proteins and a subtype specific module that in most cases contains at least one RAMP (Fig. 1A). An additional subtype (Cas module RAMP, $\mathrm{Cmr}$ ) includes many RAMP proteins and a polymerase/ nuclease. This system seems to share core Cas proteins with another subtype that resides on the same genome. As discussed later, the Cmr cluster at least to some extent, resembles the Csm-subtype. The distribution of the closely related subtypes in phylogenetically distant organisms, suggests that the CRISPR/Cas system has frequently been exchanged by horizontal gene transfer between distant micro-organisms (Makarova et al. 2006; Horvath et al. 2009). This hypothesis is supported by the observation that the CRISPR/Cas can be located on plasmids (e.g., the megaplasmids from Thermus thermophilus). Other plasmids have been reported to contain a CRISPR locus without associated cas genes (e.g., the pNOB8 conjugative plasmid from Sulfolobus solfataricus) (Godde and Bickerton 2006).

The best conserved cas genes are cas 1 and cas 2 that are present in all subtypes (Haft et al. 2005). Therefore, they are suitable markers for the presence of CRISPR/Cas. The putative nuclease/integrase Cas1 (Makarova et al. 2006) has been demonstrated to be a metal dependent nuclease that cleaves ssDNA and dsDNA, generating $\sim 80$ bp DNA fragments from dsDNA. The Cas1 structure reveals a novel fold with a two-domain architecture (Wiedenheft et al. 2009). The small Cas 2 protein cleaves ssRNAs in U-rich regions. Crystal structures of Cas2 from several species have been solved, revealing a ferredoxin fold, which is not common for endoribonucleases (Beloglazova et al. 2008). Cas1 has been proposed to be involved in spacer integration (Makarova et al. 2006), a prediction that is in agreement with the observation that Cas1 and Cas2 in E. coli are not involved in the antiviral defense stage of the mechanism when a spacer is already present in the CRISPR array (Brouns et al. 2008; Hale et al. 2009). Fusion of cas1 and cas4 genes in several genomes, including that of Geobacter sulfurreducens, suggests that Cas4, a putative RecB-like nuclease (Makarova et al. 2006), might also be involved in spacer acquisition (van der Oost et al. 2009). Cas3 is a special case, typically being a single polypeptide composed of two domains: an HD domain that has metal-dependent nuclease activity on double-stranded oligonucleotides (Aravind and Koonin 1998; Han and Krauss 2009) and a DEAD/H box helicase domain (Makarova et al. 2006). Interestingly, in the Csa-subtype the domains are separated, and in the Csy-subtype Cas3 is fused to Cas2 (Makarova et al. 2006). Cas5 and Cas6, previously annotated as core Cas proteins as well, represent a group of distantly related Cas proteins referred to as RAMPs. They appear to have similar 3D structures, and share at least a carboxy-terminal glycine-rich loop (Makarova et al. 2002). Two RAMP proteins (CasE and Cas6) have recently been demonstrated to be metal-independent endonucleases involved in the processing of CRISPR RNA (pre-crRNA), as described later (Brouns et al. 2008; Carte et al. 2008). Additionally, two types of multisubunit Cas complexes have recently been characterized. In E. coli, a complex is encoded by five clustered genes cse $1-4$ and cas5e (Cas5e and Cse 3 are RAMPs) and the gene products form a Cse-complex termed Cascade (CRISPR-associated complex for antiviral defense) (Brouns et al. 2008). A crRNA-binding Comr-complex comprising Cmr1-6 has been isolated from Pyrococcus furiosus (Hale et al. 2009). An overview of experimentally determined and putative activities and structures of core Cas proteins and Cas complexes is provided in Table 1.

\section{MODE OF ACTION}

The CRISPR/Cas mechanism can be divided into three distinct stages. The first stage concerns the integration of nucleic acid fragments of invading mobile genetic elements as new spacers into the CRISPR locus. In the second stage, the CRISPR is transcribed as a precursor (pre-crRNA), which is subsequently cleaved by a dedicated endoribonuclease, resulting in mature crRNAs that remain associated 
Table 1. Cas proteins involved in different stages.*

\begin{tabular}{|c|c|c|c|}
\hline $\begin{array}{l}\text { Stage } \\
\text { involved }\end{array}$ & Name & Activity & Remarks \\
\hline \multirow[t]{3}{*}{ Acquisition } & Cas 1 & $\begin{array}{l}\text { DNA endonuclease (Wiedenheft } \\
\text { et al. 2009), RNA and DNA } \\
\text { binding (Han et al. 2009) }\end{array}$ & Crystal structures (Wiedenheft et al. 2009) (3GOD and 2YZS) \\
\hline & Cas2 & $\begin{array}{l}\text { Ribonuclease activity } \\
\text { (Beloglazova et al. 2008) }\end{array}$ & $\begin{array}{l}\text { Crystal structures (Beloglazova et al. 2008) (2I8E, 1ZPW, 2IOX, and } \\
\text { 2IVY) }\end{array}$ \\
\hline & Cas4 & & RecB-like nuclease \\
\hline Processing & $\begin{array}{l}\text { CasE ( part of } \\
\text { Cascade), Cas6 }\end{array}$ & $\begin{array}{l}\text { pre-crRNA cleavage (Brouns et al. } \\
\text { 2008; Carte et al. 2008) }\end{array}$ & $\begin{array}{l}\text { Crystal structures of CasE (Ebihara et al. 2006) (1WJ9) and Cas6 } \\
\quad(\text { Carte et al. 2008) (3I4H) }\end{array}$ \\
\hline \multirow[t]{3}{*}{ Interference } & $\begin{array}{l}\text { Cse-complex } \\
\quad \text { (Cascade) }\end{array}$ & & $\begin{array}{l}\text { Comprises CasA-E. Crystal structures solved of CasB (Agari et al. } \\
\text { 2008) (2ZCA) and CasE (see earlier discussion) }\end{array}$ \\
\hline & Cas3 & $\begin{array}{l}\text { Nuclease activity of HD domain } \\
\quad \text { (Han and Krauss 2009) }\end{array}$ & $\begin{array}{l}\text { Helicase, often fused to HD-domain (Makarova et al. 2006). } \\
\text { Possibly also involved in acquisition, according to fusion to Cas2 } \\
\text { in Csy-subtype (van der Oost et al. 2009) }\end{array}$ \\
\hline & Cmr-complex & $\begin{array}{l}\text { Cleavage of RNA complementary } \\
\text { to crRNA (Hale et al. 2008) }\end{array}$ & $\begin{array}{l}\text { Comprises Cmr1-6. Two structures of Cmr5 are available } \\
\text { (Sakamoto et al. 2009) (2OEB and 2ZOP) }\end{array}$ \\
\hline
\end{tabular}

*Experimentally determined activities and PDB ID codes are indicated.

with a Cas protein complex. During the third and final stage, the crRNA guides the Cas complex to known, invading nucleic acids to neutralize the invader, most likely by cleavage.

\subsection{Integration of New Spacers}

The first experimental evidence that the CRISPR/Cas system is indeed an antiviral defense system was obtained from phage infection experiments of the lactic acid bacterium Streptococcus thermophilus that has a CRISPR/Cas locus of the Csn-subtype (Fig. 1A) (Barrangou et al. 2007; Deveau et al. 2008; Horvath et al. 2008). Screening for adaptation of the CRISPR locus in the surviving bacteria revealed that a subpopulation of survivors had acquired new phage-specific spacer sequences (Fig. 2). Subsequent deletion of these new spacer sequences resulted in loss of the acquired resistance, demonstrating the correlation of spacer presence and phage resistance (Barrangou et al. 2007). Comparative analysis of the spacer-targeted region of the viral genome revealed a sequence motif called CRISPR motif or proto-spacer adjacent motif (PAM) (i.e., NNAGAAW) downstream of the proto-spacer. The phages responded to the resistance of the host by mutations in the proto-spacer, but also by mutations in the motif (Deveau et al. 2008), illustrating the constant battle between phages and bacteria. Spacers that did not have a perfect PAM were integrated as well, but these were always accompanied by spacers with a perfect PAM (Deveau et al. 2008), the latter probably being essential for resistance. The data suggests that the PAM is crucial for target interference, but not for integration of new spacers. Genomic analysis of proto-spacers revealed the presence of slightly different
PAMs in many extrachromosomal elements, and the conservation of PAM sequences correlates well with the CRISPR repeat types, and thus cas gene subtypes (Mojica et al. 2009).

The spacers in S. thermophilus were integrated at the leader proximal end of the CRISPR locus, suggesting that a CRISPR array is a chronological record of past phage infections. The role of the leader sequence is not exactly known, but it is possibly required for repeat duplication and/or spacer integration. Polarity of the CRISPR integration had been previously predicted by Lillestol and coworkers after comparing the CRISPRs of two Sulfolobus solfataricus strains with major variation at the leader-side

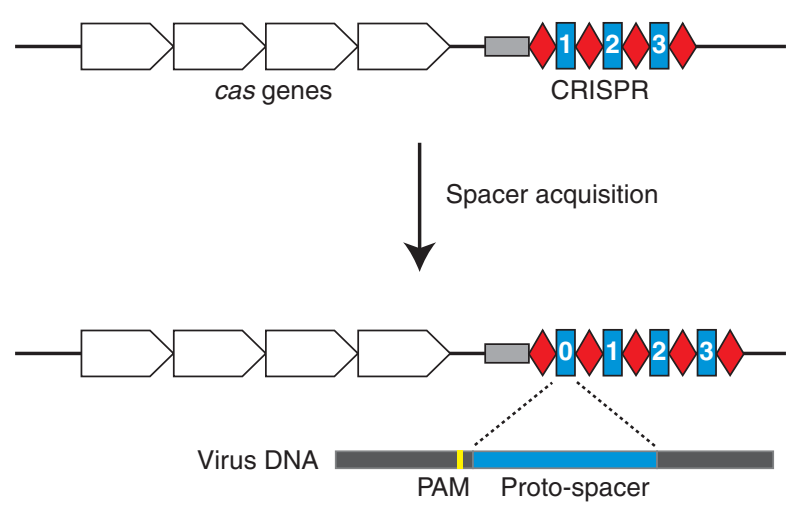

Figure 2. Integration of a new spacer. A new spacer is acquired at the leader proximal side of the CRISPR during virus infection, resulting in resistance. The CRISPR consist of a leader (grey box), repeats (red diamonds), and spacers (blue boxes). The newly acquired spacer is numbered 0 and matches the sequence of the virus (proto-spacer). The proto-spacer adjacent motif (PAM) is located downstream or upstream the proto-spacer. 
of the CRISPR (Lillestol et al. 2006). Next to acquired resistance by spacer integration in $S$. thermophilus, the above mentioned study also showed that cas genes are involved in CRISPR-mediated defense. Disruption of the $\operatorname{csn} 1$ gene resulted in loss of viral resistance. An interrupted csn 2 gene did not lead to loss of resistance, but rather to a disrupted ability to integrate new spacers. This does not only show that both cas genes are involved in resistance, but also that they play a role at different stages. Csn2 is apparently needed for integration of new spacers, but it is restricted to the Csn-subtype. In Wollinella succinogenes, which carries the same subtype, $\operatorname{csn} 2$ is absent and seems to be replaced by cas4 (van der Oost et al. 2009). Thus, despite the unknown role of Csn2 in spacer integration, it is likely that it is replaced by Cas proteins with analogous functions in other Cas subtypes, Cas4 being a likely candidate. Csn1 may have a function analogous to Cascade/Cas3 (as discussed later, Cas3 is also involved in target interference) (van der Oost et al. 2009).

\subsection{CRISPR Transcription and Processing}

Before it was hypothesized that the CRISPR/Cas system protects the host against invading nucleic acids, studies on short RNAs in the archaea Archaeoglobus fulgidus and S. solfataricus had already indicated that CRISPRs are actively transcribed and the pre-crRNA processed (Tang et al. 2002; Tang et al. 2005). Although studies on transcription and processing in S. solfataricus showed that transcription can be bidirectional (Lillestol et al. 2006; Lillestol et al. 2009), most other studies have reported unidirectional transcription from the leader proximal side (Brouns et al. 2008; Hale et al. 2008; Marraffini and Sontheimer 2008; Semenova et al. 2009). It is therefore anticipated that in most cases a single promoter at the leader side controls transcription of a CRISPR locus. It has recently been shown that in the case of E. coli $\mathrm{K} 12$ the promoter indeed resides in the leader region (Pul et al. 2010). CRISPR transcription and processing has been studied in more detail for this $E$. coli K12 system, which consists of 8 cas genes upstream of a CRISPR (Fig. 1A). The Cas proteins were overexpressed in $E$. coli BL21, a strain that lacks endogenous cas genes (Studier et al. 2009). Pull down analysis revealed the presence of Cascade, a protein complex that contains five different subunits, CasABCDE. Northern blot analysis of crRNAs in E.coli K12 revealed short RNAs, the size of which corresponded to approximately one spacer and one repeat. Omitting the cas genes one-by-one identified CasE, a RAMP protein, as a potential candidate for pre-crRNA processing. This finding was confirmed by in vitro activity assays, showing that CasE is a metal-independent endoribonuclease that specifically cleaves a precursor (pre-crRNA) into mature crRNAs. After processing, these crRNAs remain tightly bound to the Cascade complex. Cloning and subsequent sequence analysis revealed more crRNA products derived from the leader proximal end of the CRISPR (Brouns et al. 2008). Independent analysis of crRNA from $P$. furiosus also uncovered more products from the leader proximal side of the CRISPR, and fewer from the distal end. This can be explained by premature termination of CRISPR transcription (Hale et al. 2008). Sequence analysis of cloned E. coli crRNA products revealed that the product contains eight nucleotides of the repeat termed the $5^{\prime}$ handle, the spacer, and a large part of the next repeat including a part of the stem-loop termed the $3^{\prime}$ handle (Brouns et al. 2008). It has been proposed that these handles, the conserved parts of the crRNAs, are bound by subunits of Cascade (Brouns et al. 2008). Based on sequence conservation in CasE and the crystal structure of a CasE homolog from Thermus thermophilus (Ebihara et al. 2006), the histidine residue at position 20 has been predicted to be a residue involved in endonuclease activity. Indeed, the activity was lost when the histidine was substituted by an alanine. In vivo analysis showed that the mutation resulted in loss of resistance, thus showing that pre-crRNA cleavage is a mechanistic requirement.

Despite the fact that Cas6 from P. furiosus shares low sequence identity (except for the carboxy-terminal glycine-rich loop, a common feature of RAMP proteins), its structure, a duplicated ferredoxin fold, is surprisingly similar to CasE from T. thermophilus (Fig. 3) (van der Oost et al. 2009). Like CasE, Cas6 displays metal independent endoribonuclease activity (Carte et al. 2008). Although the folded secondary structure of the repeat RNA of P. furiosus is debatable (Kunin et al. 2007), its crRNA cleavage product also contains an eight-nucleotide $5^{\prime}$ handle (psi-tag) (Fig. 1B and C). Unlike the proposed single histidine site in CasE (Brouns et al. 2008), a potential catalytic triad with a histidine, tyrosine, and a lysine residue is present in Cas6 (Fig. 3) (Carte et al. 2008). This predicted catalytic site is structurally similar to that of tRNA splicing enzymes. Analysis of the cleaved pre-crRNA products revealed that cleavage occurs at the $3^{\prime}$ side of the phosphodiester bond, generating a $5^{\prime}$ end hydroxyl group and a $2^{\prime}, 3^{\prime}$ end cyclic phosphate group, analogous to tRNA splicing enzymes (Calvin and Li 2008; Carte et al. 2008). Both CasE and Cas6 probably cleave the pre-crRNA following a general acid-base hydrolysis mechanism (Fig. 3c). Besides its endoribonucleolytic activity, Cas6 binds the $3^{\prime}$ handle of the crRNA. However, in P. furiosus, the endonucleolytic product is further trimmed to active mature crRNAs lacking a $3^{\prime}$ handle (Hale et al. 2008; Hale et al. 2009), whereas mature crRNAs in E. coli do contain a $3^{\prime}$ handle. Another difference is the fact that CasE remains part of the Cascade 
A

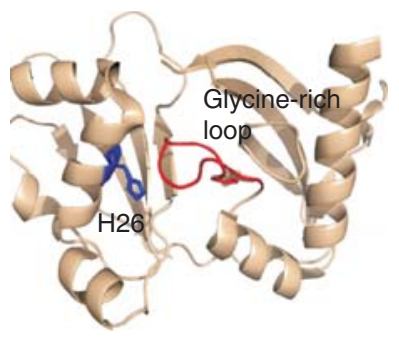

B

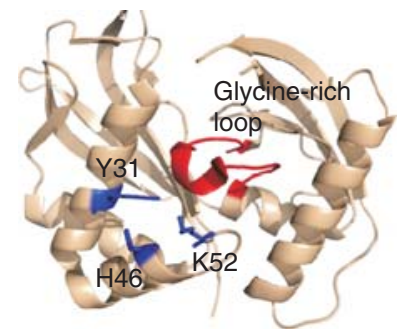

C

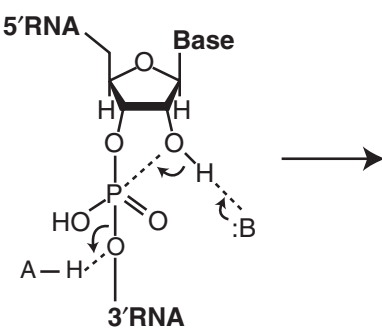

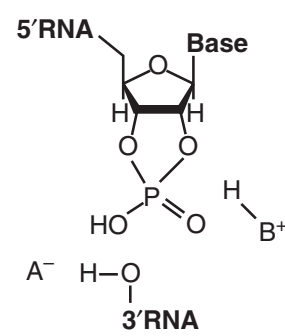

Figure 3. The catalytic sites of CasE and Cas6, and the proposed reaction mechanism of pre-crRNA cleavage. (A) Proposed catalytic site of CasE from T. thermophilus showing the conserved histidine residue (H26) and the glycine-rich carboxy-terminal loop. The catalytic site of Cas6 from $P$. furiosus $(B)$ contains a catalytic triad of tyrosine (Y31), histidine (H46) and lysine (K52) and a glycine-rich carboxyterminal loop. The loop and the overall duplicated ferredoxin fold are conserved among CasE and Cas6. Pre-crRNA cleavage might follow a general acid-base hydrolysis mechanism $(C)$. A base (B) draws a proton from the $2^{\prime} \mathrm{OH}$ of the ribose ring. A subsequent nucleophilic attack on the phosphorus atom is simultaneously compensated by the acid (A) that donates a proton to the leaving $3^{\prime}$ RNA. The tyrosine residue of Cas 6 is proposed to be the base and the histidine the acid residue (Carte et al. 2008). In CasE the histidine and a water molecule might be the catalytic residues. Pictures in $(A)$ and $(B)$ are generated with pymol (www.pymol.org), potential catalytic residues are depicted in blue; the glycine-rich loop is depicted in red. Coordinates were obtained from the Protein Data Bank (www.pdb.org).

complex (Brouns et al. 2008), whereas Cas6 has not been identified as part of the pyrococcal Cmr-complex (see the following) (Hale et al. 2009); apparently cleaved crRNAs in P. furiosus are transferred to the Cmr-complex. Besides the Cmr-complex, an additional Cst-complex might be present in $P$. furiosus, that is encoded by three cst genes downstream of the $\mathrm{cmr}$ module (Fig. 1A). Overall, it has become clear that despite some mechanistic similarity, also substantial differences exists between the different CRISPR/Cas subtypes.

\subsection{Target Interference}

Although it was initially hypothesized that the CRISPR/ Cas system would target alien RNA, analogous to RNAi (Makarova et al. 2006), several studies have indicated that the target of the CRISPR system rather is invading DNA.

The first observation supporting this hypothesis was made in virus infection studies with $S$. thermophilus, revealing that spacer sequences corresponding to either the coding or noncoding strand were integrated (Barrangou et al. 2007). The CRISPR locus has been demonstrated to be transcribed only from the leader proximal side in E. coli, Staphyloccuus epidermidis and P. furiosus (Brouns et al. 2008; Hale et al. 2008; Marraffini and Sontheimer 2008); to date the only exception appears to be the case of S. solfataricus (Lillestol et al. 2006; Lillestol et al. 2009). A consequence of monodirectional CRISPR transcription would be that generated crRNAs have to be complementary to the mRNA of the virus. Only spacers from one strand should be incorporated in case of an antisense RNA mechanism. The observation that this is generally not the case suggests that DNA is the target. Additional evidence that DNA is the target was provided by a study on an engineered E. coli Cas system in which artificial spacers were unidirectionally transcribed. Generating crRNAs complementary to both the coding strand and the template strand were successful in inhibiting virus proliferation (Brouns et al. 2008). Furthermore, a study on plasmid conjugation in S. epidermidis convincingly proved that DNA is being targeted (Marraffini and Sontheimer 2008). A natural spacer from the CRISPR of $S$. epidermidis has a perfect match with a gene of a conjugative plasmid. This spacer confers resistance and prevents conjugation of this plasmid. A selfsplicing intron was inserted into the center of the protospacer sequence in the plasmid. After conjugation and subsequent transcription, this intron is spliced, generating a mature mRNA that contains a fully complementary sequence to the crRNA. The plasmid was able to escape the CRISPR/Cas system, showing that mRNA is not being targeted; hence, the target must be DNA that could not be recognized by the crRNA because of the intron DNA sequence interrupting the proto-spacer. This finding is confirmed by an additional experiment in which a fragment of the targeted gene is inserted in a plasmid, in both orientations. The fragment is not essential for propagation of the plasmid. Nevertheless, the inserted fragment, in both orientations, dramatically decreased the transformation efficiencies, again indicating that DNA is being targeted. Recently, an exceptional CRISPR/Cas system has been described, i.e., the Cmr-subtype from $P$. furiosus that appears to target and degrade RNA (discussed later). For this system, however, no natural targets have been found yet.

Most biochemical and mechanistic information on the pre-crRNA and target interference is derived from the earlier mentioned E. coli and P. furiosus model systems. Requirements for CRISPR-based resistance were determined in E. coli BL21. Artificial CRISPRs were designed to target four different genes of phage lambda. Overexpression of 
Cascade and CRISPR RNA was sufficient to yield mature crRNAs that were bound and protected by Cascade (Brouns et al. 2008). No resistance was observed when the CRISPRs targeting phage lambda were coexpressed with Cascade only. However, coexpression of this crRNA-loaded Cascade with the Cas3 protein did result in a dramatic increase of resistance towards phage lambda infection. The role of the two-domain protein Cas3 remains to be elucidated, but it is tempting to speculate that the HD nuclease domain cleaves targeted DNA. This Cse-subtype from E. coli K12 targets DNA, but how does it discriminate between self DNA (namely the CRISPR that contains a spacer complementary to the crRNA) and nonself DNA (the invader)? A recent study in S. epidermidis revealed that the flanking sequence of the proto-spacer is crucial for self versus nonself DNA recognition, and thus for interference (Marraffini and Sontheimer 2010b). It was shown that a targeted conjugative plasmid could bypass host resistance by the CRISPR/Cas system if three bases downstream of the proto-spacer were complementary to the CRISPR repeat sequence (Marraffini and Sontheimer 2010b). In other subtypes, however, the PAM might help prevent autoimmunity by being present in the targeted invading DNA sequence, and absent from the CRISPR DNA sequence. The PAM determines if invading nucleic acids are being targeted in the case of $S$. thermophilus, as can be deduced from phages that have mutated their PAM sequence and thus can escape the CRISPR/Cas system (Deveau et al. 2008). The two mechanisms of self versus non-self DNA recognition appear to be fundamentally different. In S. epidermidis the potential of the downstream sequence of the proto-spacer to basepair with the CRISPR repeat determines whether the DNA is being targeted, while in, for example, S. thermophilus the PAM determines whether the DNA is being targeted. To bypass immunity in S. epidermidis, the invader can only mutate its flanking nucleic acids to a sequence complementary to the repeat DNA, while in S. thermophilus, the invader can mutate its PAM to any other sequence.

Interestingly, the Cas-system from P. furiosus has recently been reported to be capable of interfering with target RNA rather than DNA (Hale et al. 2009). With native Northern blot analysis, a Cmr-type protein complex was identified that forms a stable interaction of crRNAs (psiRNA) (Hale et al. 2008; Hale et al. 2009). The crRNAs are 39 and 45 nucleotides in length, containing an identical $5^{\prime}$ handle but different $3^{\prime}$ ends. The RNP complex isolated from Pyrococcus comprised six distinct Cas proteins: Cmr1-6. Cas6 was not part of the complex (Hale et al. 2009), unlike its functional analog CasE that is a core subunit of the Cascade complex (Cse-complex) in E. coli (Brouns et al. 2008). The isolated RNP complex cleaved complementary RNA but not ssDNA. The 39 and 45 nucleotide-long crRNAs resulted in two different cleavage sites in the target mRNA. Both cleaved 14 nucleotides upstream from the $3^{\prime}$ end of the crRNA, suggesting a molecular ruler mechanism for cleavage (Fig. 4B). Reconstitution of the RNP complex from purified subunits revealed that all except Cmr5 are essential for target RNA cleavage (Hale et al. 2008; Hale et al. 2009). The Cmr2 protein that is part of the Cmr-complex contains a PALM (polymerase) domain, fused to a HD-nuclease domain. Whereas the biological function of the polymerase is not known, the HD-nuclease might be responsible for degradation of the target RNA. At least two proteins from the Cmr-complex are related to proteins that are encoded by the $c s m$ module in S. epidermidis (Fig. 1A). The polymerase Cmr2 is related to Csm1 and the RAMP protein Cmr4 is related to Csm3 (Haft et al. 2005). The Csm-type system from S. epidermidis has been demonstrated to target DNA in vivo, which is in contrast to the observed in vitro RNA cleavage activity of the Cmr-complex. Future biochemical and in vivo analyses are required to resolve this apparent contradiction.

\section{ANALOGY WITH RNAi IN EUKARYOTES}

The function and biogenesis of crRNAs and the mechanism of target interference display striking analogies with small regulatory RNAs in eukaryotes. The eukaryotic regulatory RNAs can be divided into three major groups: endogenous miRNAs that generally silence host gene expression, piRNAs that silence transposable elements in animal germ cells, and siRNAs that can be involved in viral RNA silencing. In short, siRNAs are derived from dsRNA that is randomly cleaved by Dicer, generating fragments with a $3^{\prime}$ dinucleotide overhang. Dicer subsequently binds the overhang and cleaves $\sim 20$ bases away from the first cleavage site following a molecular ruler mechanism, generating short dsRNAs with $3^{\prime}$ overhangs and $5^{\prime}$ phosphates (Bernstein et al. 2001; Macrae et al. 2006; MacRae and Doudna 2007). These dsRNAs are transferred to the Argonaute protein in the RNA induced silencing complex (RISC) that also consists of Dicer and a dsRNA binding protein; the latter determines in which orientation the dsRNA molecule is loaded onto Argonaute (Tomari et al. 2004). Argonaute recognizes the passenger strand and degrades it, retaining the guide strand (Rand et al. 2005). When RISC encounters a perfectly complementary RNA, it can interact with it by Watson-Crick base pairing. Argonaute subsequently cleaves the complementary target RNA strand, 10 nucleotides away from the $5^{\prime}$ end of the guide, after which the target fragments are released and the RISC complex is recycled for a new target degradation event, eventually resulting in silencing the virus (Baulcombe 2004) (Fig. 4C). 
A

B

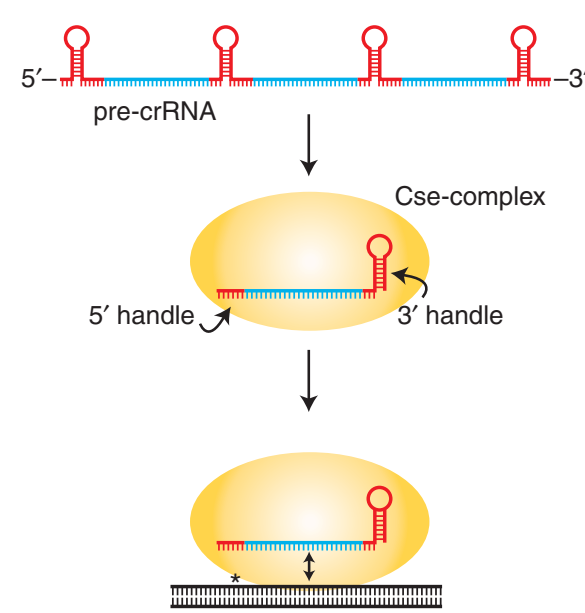

Target DNA
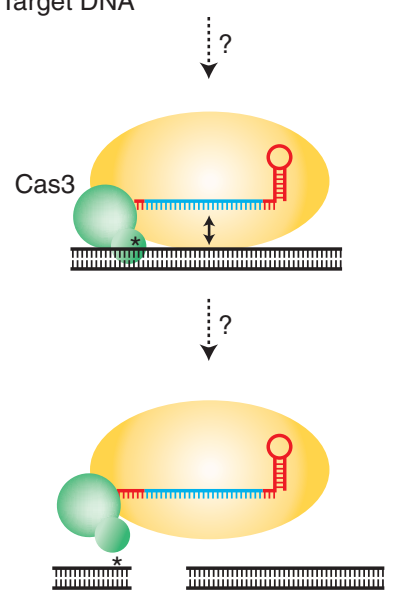
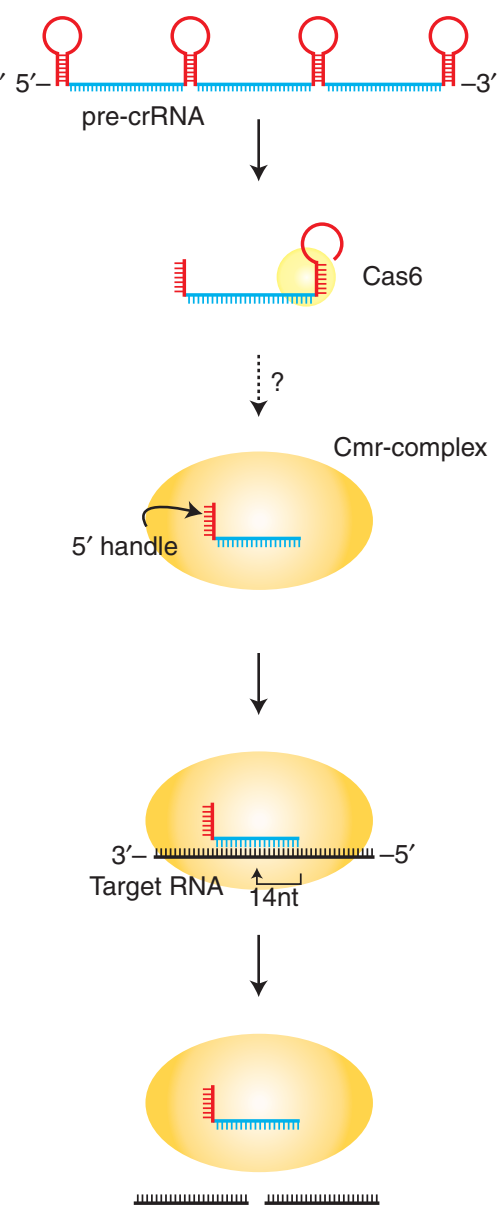

C

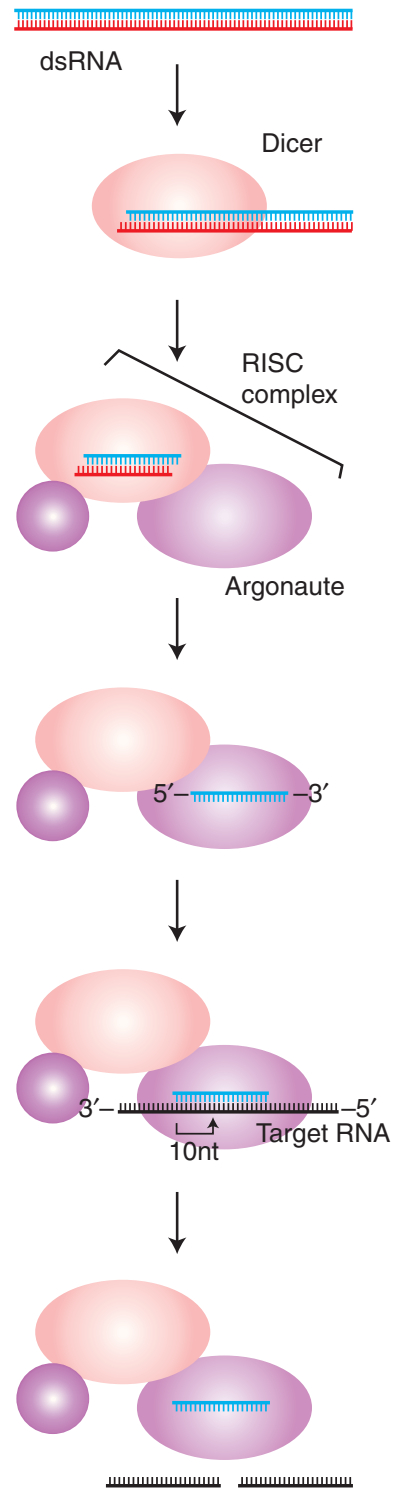

Figure 4. Antiviral DNA and RNA silencing pathways in prokaryotes and eukaryotes. $(A)$ crRNA mediated DNA silencing pathway in E. coli. pre-crRNA is cleaved by the CasE subunit of Cascade (Cse-complex) and the mature crRNA remains bound to Cascade. How Cascade, assisted by Cas3, recognizes and neutralizes invading DNA remains to be elucidated. $(B)$ crRNA mediated RNA silencing pathway in $P$. furiosus. Pre-crRNA is cleaved by Cas6 and then further trimmed to generate crRNAs of two different lengths. These crRNAs are bound by the Cmr-complex. This loaded Cmr-complex specifically binds viral RNA and cleaves the complementary strand 14 nucleotides away from the $3^{\prime}$ end of the crRNA. This pathway shares functional analogies with siRNA mediated antiviral resistance in eukaryotes. $(C)$ siRNAs are generated from viral dsRNA by dicer. The first (random) cleavage event by dicer generates dsRNA with a $3^{\prime}$ dinucleotide overhang. The second cleavage by dicer takes place $20-25$ bases away from the overhang, generating short dsRNAs. The dsRNA is transferred to the Argonaute protein of the RISC complex and the passenger strand is removed. The retained guide strand can basepair with a complementary viral mRNA molecule, followed by a cleavage of the scissile bond between the 10th and 11th base from the $3^{\prime}$ end of the guide strand. The cleaved target RNA dissociates and the recycled RISC can be used in a second round of RNA binding and cleavage. Please note that dashed arrows indicate processes that are based on hypotheses. 
Although sequence comparison of the prokaryotic CRISPR/Cas and the eukaryotic siRNA system indicates they are phylogenetically unrelated (Makarova et al. 2006), they share some functional and mechanistic analogies. Both crRNA and siRNA are derived from large RNA precursors. Furthermore, both the Cmr-complex from $P$. furiosus and RISC complex specifically bind their target RNA by base pairing and degrade it by a molecular ruler mechanism. The major difference between the two systems is that crRNAs are transcribed from the host chromosome and are part of an adaptive immune system, whereas antiviral siRNAs are derived from the invaders and are part of an innate immune system. Moreover, the Cse- and Csm-type CRISPR/Cas systems target DNA rather than RNA. The RNAi pathways in which Dicer and Argonaute are the key players are restricted to eukaryotes. The only conserved component among prokaryotes and eukaryotes is the Argonaute protein. The ones from prokaryotes have been useful models in crystallization studies (Jinek and Doudna 2009; Wang et al. 2009). It has recently been hypothesized that prokaryotic Argonaute proteins are involved in yet another DNA-mediated antiviral defense mechanism (Makarova et al. 2009).

\section{CONCLUDING REMARKS}

The adaptive and heritable antiviral and antiplasmid CRISPR/Cas system described here is of particular interest because of potential applications in research (silencing of genes in prokaryotes), in medical diagnostics (strain typing by comparison of CRISPR spacer sequences), and industry (development of phage resistant strains) (Sorek et al. 2008). Although the rapidly expanding research field on CRISPR/ Cas yields a steady gain of insight, many questions on this fascinating defense system still remain to be answered. One poorly understood aspect is the mechanism of spacer integration. A prime candidate to be involved in this process is the Cas1 protein, because it is very well conserved among different subtypes (Jansen et al. 2002; Haft et al. 2005; Makarova et al. 2006; Marraffini and Sontheimer 2009), it has nuclease activity (Wiedenheft et al. 2009), and it is not necessary for crRNA processing or target interference (Brouns et al. 2008). Lactic acid bacteria such as S. thermophilus seem to be the best organisms to study this process because it is the only class of organisms to date where spacer integration has been observed in laboratory experiments (Barrangou et al. 2007). Significant advances have been made as far as CRISPR transcription and processing are concerned. Recently, a possible regulator, Heat-stable Nucleoid Structuring protein (H-NS), has been identified in E. coli K12. This regulator possibly represses transcription of the CRISPR and cascade genes by binding to their promoter regions
(Pul et al. 2010). This poses an interesting lead for future research on how the system is regulated, although this likely differs substantially in other organisms. The biogenesis of crRNAs has been unraveled in more detail but it is unknown how these mature crRNAs guide the Cas protein machinery to their complementary target. Some first light has been shed on how the Cmr-subtype of P. furiosus degrades complementary RNA. But how other subtypes, such as the Csm- and Cse-subtype, trace and target complementary incoming DNA remains to be answered. Major breakthroughs with answers to these questions are to be expected in the near future.

\section{ACKNOWLEDGMENTS}

The authors are financially supported by a Vici grant to J.v.d.O. and a Veni grant to S.J.J.B. from the Dutch Organisation for Scientific Research (Nederlandse Organisatie voor Wetenschappelijk Onderzoek).

\section{REFERENCES}

Agari Y, Yokoyama S, Kuramitsu S, Shinkai A. 2008. X-ray crystal structure of a CRISPR-associated protein, Cse2, from Thermus thermophilus HB8. Proteins 73: 1063-1067.

Andersson AF, Banfield JF. 2008. Virus population dynamics and acquired virus resistance in natural microbial communities. Science 320: $1047-1050$.

Aravind L, Koonin EV. 1998. The HD domain defines a new superfamily of metal-dependent phosphohydrolases. Trends Biochem Sci 23: $469-472$.

Banfield JF, Young M. 2009. Microbiology. Variety-the splice of life-in microbial communities. Science 326: 1198-1199.

Barrangou R, Fremaux C, Deveau H, Richards M, Boyaval P, Moineau S, Romero DA, Horvath P. 2007. CRISPR provides acquired resistance against viruses in prokaryotes. Science 315: 1709-1712.

Baulcombe D. 2004. RNA silencing in plants. Nature 431: 356-363.

Beloglazova N, Brown G, Zimmerman MD, Proudfoot M, Makarova KS, Kudritska M, Kochinyan S, Wang S, Chruszcz M, Minor W, et al. 2008. A novel family of sequence-specific endoribonucleases associated with the clustered regularly interspaced short palindromic repeats. J Biol Chem 283: 20361-20371.

Bergh O, Borsheim KY, Bratbak G, Heldal M. 1989. High abundance of viruses found in aquatic environments. Nature 340: 467-468.

Bernstein E, Caudy AA, Hammond SM, Hannon GJ. 2001. Role for a bidentate ribonuclease in the initiation step of RNA interference. Nature 409: $363-366$.

Bolotin A, Quinquis B, Sorokin A, Ehrlich SD. 2005. Clustered regularly interspaced short palindrome repeats (CRISPRs) have spacers of extrachromosomal origin. Microbiology 151: 2551-2561.

Brouns SJ, Jore MM, Lundgren M, Westra ER, Slijkhuis RJ, Snijders AP, Dickman MJ, Makarova KS, Koonin EV, van der Oost J. 2008. Small CRISPR RNAs guide antiviral defense in prokaryotes. Science 321: 960-964.

Calvin K, Li H. 2008. RNA-splicing endonuclease structure and function. Cell Mol Life Sci 65: 1176-1185.

Carte J, Wang R, Li H, Terns RM, Terns MP. 2008. Cas6 is an endoribonuclease that generates guide RNAs for invader defense in prokaryotes. Genes Dev 22: 3489-3496.

Chopin MC, Chopin A, Bidnenko E. 2005. Phage abortive infection in lactococci: variations on a theme. Curr Opin Microbiol 8: 473-479. 
Deveau H, Barrangou R, Garneau JE, Labonte J, Fremaux C, Boyaval P, Romero DA, Horvath P, Moineau S. 2008. Phage response to CRISPR-encoded resistance in Streptococcus thermophilus. J Bacteriol 190: $1390-1400$.

Ebihara A, Yao M, Masui R, Tanaka I, Yokoyama S, Kuramitsu S. 2006. Crystal structure of hypothetical protein TTHB192 from Thermus thermophilus HB8 reveals a new protein family with an RNA recognition motif-like domain. Protein Sci 15: 1494-1499.

Godde JS, Bickerton A. 2006. The repetitive DNA elements called CRISPRs and their associated genes: evidence of horizontal transfer among prokaryotes. J Mol Evol 62: 718-729.

Haft DH, Selengut J, Mongodin EF, Nelson KE. 2005. A guild of 45 CRISPR-associated (Cas) protein families and multiple CRISPR/ Cas subtypes exist in prokaryotic genomes. PLoS Comput Biol 1: e60.

Hale C, Kleppe K, Terns RM, Terns MP. 2008. Prokaryotic silencing (psi)RNAs in Pyrococcus furiosus. RNA 14: 2572-2579.

Hale CR, Zhao P, Olson S, Duff MO, Graveley BR, Wells L, Terns RM, Terns MP. 2009. RNA-guided RNA cleavage by a CRISPR RNA-Cas protein complex. Cell 139: 945-956.

Han D, Krauss G. 2009. Characterization of the endonuclease SSO2001 from Sulfolobus solfataricus P2. FEBS Lett 583: 771-776.

Han D, Lehmann K, Krauss G. 2009. SSO1450-a CAS1 protein from Sulfolobus solfataricus P2 with high affinity for RNA and DNA. FEBS Lett 583: $1928-1932$.

Heidelberg JF, Nelson WC, Schoenfeld T, Bhaya D. 2009. Germ warfare in a microbial mat community: CRISPRs provide insights into the co-evolution of host and viral genomes. PLoS One 4: e4169.

Held NL, Whitaker RJ. 2009. Viral biogeography revealed by signatures in Sulfolobus islandicus genomes. Environ Microbiol 11: 457-466.

Hofnung M, Jezierska A, Braun-Breton C. 1976. lamB mutations in E. coli K12: growth of $\lambda$ host range mutants and effect of nonsense suppressors. Mol Gen Genet 145: 207-213.

Horvath P, Barrangou R. 2010. CRISPR/Cas, the immune system of bacteria and archaea. Science 327: 167-170.

Horvath P, Coute-Monvoisin AC, Romero DA, Boyaval P, Fremaux C, Barrangou R. 2009. Comparative analysis of CRISPR loci in lactic acid bacteria genomes. Int J Food Microbiol 131: 62-70.

Horvath P, Romero DA, Coute-Monvoisin AC, Richards M, Deveau H, Moineau S, Boyaval P, Fremaux C, Barrangou R. 2008. Diversity, activity, and evolution of CRISPR loci in Streptococcus thermophilus. J Bacteriol 190: 1401-1412.

Ishino Y, Shinagawa H, Makino K, Amemura M, Nakata A. 1987. Nucleotide sequence of the iap gene, responsible for alkaline phosphatase isozyme conversion in Escherichia coli, and identification of the gene product. J Bacteriol 169: 5429-5433.

Jansen R, Embden JD, Gaastra W, Schouls LM. 2002. Identification of genes that are associated with DNA repeats in prokaryotes. Mol Microbiol 43: 1565-1575.

Jinek M, Doudna JA. 2009. A three-dimensional view of the molecular machinery of RNA interference. Nature 457: 405-412.

Karginov FV, Hannon GJ. 2010. The CRISPR system: small RNA-guided defense in bacteria and archaea. Mol Cell 37: 7-19.

Kunin V, Sorek R, Hugenholtz P. 2007. Evolutionary conservation of sequence and secondary structures in CRISPR repeats. Genome Biol 8: R61.

Lillestol RK, Redder P, Garrett RA, Brugger K. 2006. A putative viral defence mechanism in archaeal cells. Archaea 2: 59-72.

Lillestol RK, Shah SA, Brugger K, Redder P, Phan H, Christiansen J, Garrett RA. 2009. CRISPR families of the crenarchaeal genus Sulfolobus: bidirectional transcription and dynamic properties. Mol Microbiol 72: 259-272.

Llosa M, Gomis-Ruth FX, Coll M, de la Cruz Fd F. 2002. Bacterial conjugation: a two-step mechanism for DNA transport. Mol Microbiol 45: $1-8$.

MacRae IJ, Doudna JA. 2007. Ribonuclease revisited: Structural insights into ribonuclease III family enzymes. Curr Opin Struct Biol 17: $138-145$.
Macrae IJ, Zhou K, Li F, Repic A, Brooks AN, Cande WZ, Adams PD, Doudna JA. 2006. Structural basis for double-stranded RNA processing by Dicer. Science 311: 195-198.

Makarova KS, Aravind L, Grishin NV, Rogozin IB, Koonin EV. 2002. A DNA repair system specific for thermophilic Archaea and bacteria predicted by genomic context analysis. Nucleic Acids Res 30: 482-496.

Makarova KS, Grishin NV, Shabalina SA, Wolf YI, Koonin EV. 2006. A putative RNA-interference-based immune system in prokaryotes: Computational analysis of the predicted enzymatic machinery, functional analogies with eukaryotic RNAi, and hypothetical mechanisms of action. Biol Direct 1: 7.

Makarova KS, Wolf YI, van der Oost J, Koonin EV. 2009. Prokaryotic homologs of Argonaute proteins are predicted to function as key components of a novel system of defense against mobile genetic elements. Biol Direct 4: 29.

Marraffini LA, Sontheimer EJ. 2008. CRISPR interference limits horizontal gene transfer in staphylococci by targeting DNA. Science 322: $1843-1845$

Marraffini LA, Sontheimer EJ. 2009. Invasive DNA, chopped and in the CRISPR. Structure 17: 786-788.

Marraffini LA, Sontheimer EJ. 2010a. CRISPR interference: RNAdirected adaptive immunity in bacteria and archaea. Nat Rev Genet 11: $181-190$.

Marraffini LA, Sontheimer EJ. 2010b. Self versus non-self discrimination during CRISPR RNA-directed immunity. Nature 463: 568-571.

Mojica FJ, Diez-Villasenor C, Garcia-Martinez J, Almendros C. 2009. Short motif sequences determine the targets of the prokaryotic CRISPR defence system. Microbiology 155: 733-740.

Mojica FJ, Diez-Villasenor C, Garcia-Martinez J, Soria E. 2005. Intervening sequences of regularly spaced prokaryotic repeats derive from foreign genetic elements. J Mol Evol 60: 174-182.

Pourcel C, Salvignol G, Vergnaud G. 2005. CRISPR elements in Yersinia pestis acquire new repeats by preferential uptake of bacteriophage DNA, and provide additional tools for evolutionary studies. Microbiology 151: 653-663.

Pul U, Wurm R, Arslan Z, Geissen R, Hofmann N, Wagner R. 2010. Identification and characterization of E. coli CRISPR-cas promoters and their silencing by H-NS. Mol Microbiol 75: 1495-1512.

Rand TA, Petersen S, Du F, Wang X. 2005. Argonaute2 cleaves the antiguide strand of siRNA during RISC activation. Cell 123: 621-629.

Sakamoto K, Agari Y, Agari K, Yokoyama S, Kuramitsu S, Shinkai A. 2009. $\mathrm{X}$-ray crystal structure of a CRISPR-associated RAMP superfamily protein, Cmr5, from Thermus thermophilus HB8. Proteins 75: 528-532.

Semenova E, Nagornykh M, Pyatnitskiy M, Artamonova II, Severinov K. 2009. Analysis of CRISPR system function in plant pathogen Xanthomonas oryzae. FEMS Microbiol Lett 296: 110-116.

Sorek R, Kunin V, Hugenholtz P. 2008. CRISPR-a widespread system that provides acquired resistance against phages in bacteria and archaea. Nat Rev Microbiol 6: 181-186.

Studier FW, Daegelen P, Lenski RE, Maslov S, Kim JF. 2009. Understanding the differences between genome sequences of Escherichia coli B strains REL606 and BL21(DE3) and comparison of the E. coli B and K-12 genomes. J Mol Biol 394: 653-680.

Sturino JM, Klaenhammer TR. 2004. Bacteriophage defense systems and strategies for lactic acid bacteria. Adv Appl Microbiol 56: 331-378.

Tang TH, Bachellerie JP, Rozhdestvensky T, Bortolin ML, Huber H, Drungowski M, Elge T, Brosius J, Huttenhofer A. 2002. Identification of 86 candidates for small non-messenger RNAs from the archaeon Archaeoglobus fulgidus. Proc Natl Acad Sci 99: 7536-7541.

Tang TH, Polacek N, Zywicki M, Huber H, Brugger K, Garrett R, Bachellerie JP, Huttenhofer A. 2005. Identification of novel non-coding RNAs as potential antisense regulators in the archaeon Sulfolobus solfataricus. Mol Microbiol 55: 469-481.

Tock MR, Dryden DT. 2005. The biology of restriction and antirestriction. Curr Opin Microbiol 8: 466-472.

Tomari Y, Matranga C, Haley B, Martinez N, Zamore PD. 2004. A protein sensor for siRNA asymmetry. Science 306: 1377-1380. 
M.M. Jore, S.J.J. Brouns, and J. van der Oost

Tyson GW, Banfield JF. 2008. Rapidly evolving CRISPRs implicated in acquired resistance of microorganisms to viruses. Environ Microbiol 10: 200-207.

van der Oost J, Jore MM, Westra ER, Lundgren M, Brouns SJ. 2009. CRISPR-based adaptive and heritable immunity in prokaryotes. Trends Biochem Sci 34: 401-407.

van der Ploeg JR. 2009. Analysis of CRISPR in Streptococcus mutans suggests frequent occurrence of acquired immunity against infection by M102-like bacteriophages. Microbiology 155: 1966-1976.
Wang Y, Juranek S, Li H, Sheng G, Wardle GS, Tuschl T, Patel DJ. 2009. Nucleation, propagation and cleavage of target RNAs in Ago silencing complexes. Nature 461: 754-761.

Wiedenheft B, Zhou K, Jinek M, Coyle SM, Ma W, Doudna JA. 2009. Structural basis for DNase activity of a conserved protein implicated in CRISPR-mediated genome defense. Structure 17: 904-912.

Wommack KE, Colwell RR. 2000. Virioplankton: Viruses in aquatic ecosystems. Microbiol Mol Biol Rev 64: 69-114. 


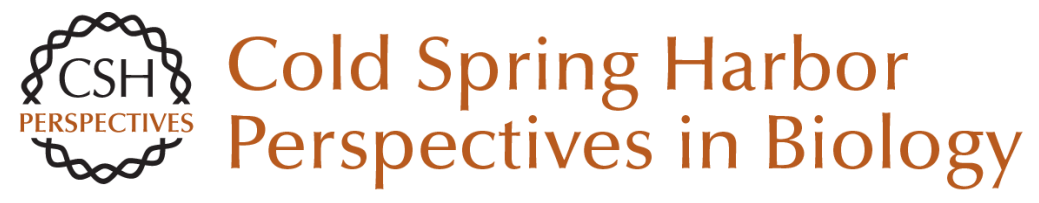

\section{RNA in Defense: CRISPRs Protect Prokaryotes against Mobile Genetic Elements}

Matthijs M. Jore, Stan J.J. Brouns and John van der Oost

Cold Spring Harb Perspect Biol 2012; doi: 10.1101/cshperspect.a003657 originally published online February 2, 2011

\section{Subject Collection RNA Worlds}

\section{Alternate RNA Structures}

Marie Teng-Pei Wu and Victoria D'Souza

Approaches for Understanding the Mechanisms

of Long Noncoding RNA Regulation of Gene

Expression

Patrick McDonel and Mitchell Guttman

Principles and Practices of Hybridization Capture

Experiments to Study Long Noncoding RNAs That

Act on Chromatin

Matthew D. Simon and Martin Machyna

Linking RNA Sequence, Structure, and Function on Massively Parallel High-Throughput

Sequencers

Sarah K. Denny and William J. Greenleaf

Extensions, Extra Factors, and Extreme

Complexity: Ribosomal Structures Provide

Insights into Eukaryotic Translation

Melanie Weisser and Nenad Ban

Nascent RNA and the Coordination of Splicing

with Transcription

Karla M. Neugebauer
Structural Biology of Telomerase
Yaqiang Wang, Lukas Susac and Juli Feigon

Structural Insights into Nuclear pre-mRNA

Splicing in Higher Eukaryotes

Berthold Kastner, Cindy L. Will, Holger Stark, et al.

What Are 3' UTRs Doing?

Christine Mayr

Single-Molecule Analysis of Reverse

Transcriptase Enzymes

Linnea I. Jansson and Michael D. Stone

\section{CRISPR Tools for Systematic Studies of RNA}

Regulation

Jesse Engreitz, Omar Abudayyeh, Jonathan

Gootenberg, et al.

Relating Structure and Dynamics in RNA Biology Kevin P. Larsen, Junhong Choi, Arjun Prabhakar, et al.

For additional articles in this collection, see http://cshperspectives.cshlp.org/cgi/collection/

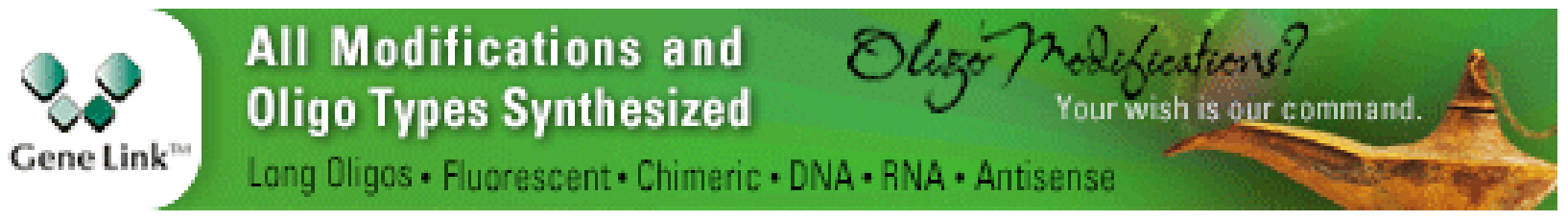

Copyright (C 2012 Cold Spring Harbor Laboratory Press; all rights reserved 
Combining Mass Spectrometry (MS) and Nuclear Magnetic Resonance (NMR) Spectroscopy for Integrative Structural Biology of Protein-RNA Complexes

Alexander Leitner, Georg Dorn and Frédéric H.-T. Allain

Discovering and Mapping the Modified Nucleotides That Comprise the Epitranscriptome of mRNA

Bastian Linder and Samie R. Jaffrey
Beyond DNA and RNA: The Expanding Toolbox of Synthetic Genetics

Alexander I. Taylor, Gillian Houlihan and Philipp Holliger

\section{Structural Basis of Nuclear pre-mRNA Splicing:} Lessons from Yeast

Clemens Plaschka, Andrew J. Newman and Kiyoshi Nagai

For additional articles in this collection, see http://cshperspectives.cshlp.org/cgi/collection/

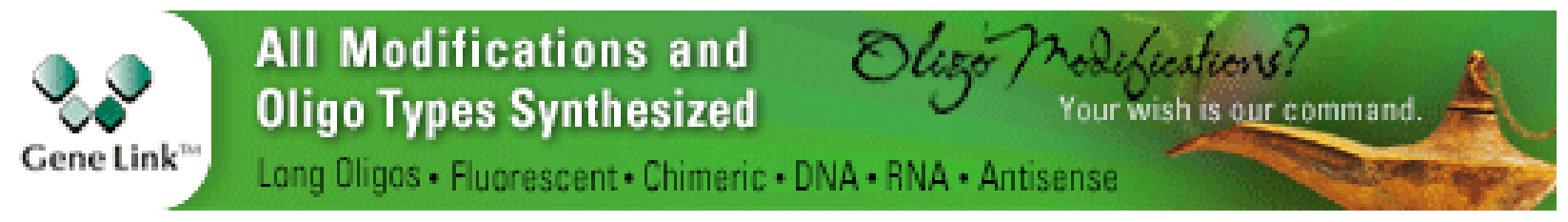

Copyright @ 2012 Cold Spring Harbor Laboratory Press; all rights reserved 